\title{
Vagal Nerve Stimulation for Treatment-Resistant Depression
}

\author{
Flavia R. Carreno ${ }^{1,2} \cdot$ Alan Frazer ${ }^{1,2,3}$
}

Published online: 5 June 2017

(C) The American Society for Experimental NeuroTherapeutics, Inc. 2017

\begin{abstract}
Major depressive disorder (MDD) is prevalent. Although standards antidepressants are more effective than placebo, up to $35 \%$ of patients do not respond to 4 or more conventional treatments and are considered to have treatmentresistant depression (TRD). Considerable effort has been devoted to trying to find effective treatments for TRD. This review focuses on vagus nerve stimulation (VNS), approved for TRD in 2005 by the Food and Drugs Administration. Stimulation is carried by bipolar electrodes on the left cervical vagus nerve, which are attached to an implanted stimulator generator. The vagus bundle contains about $80 \%$ of afferent fibers terminating in the medulla, from which there are projections to many areas of brain, including the limbic forebrain. Various types of brain imaging studies reveal widespread functional effects in brain after either acute or chronic VNS. Although more randomized control trials of VNS need to be carried out before a definitive conclusion can be reached about its efficacy, the results of open studies, carried out over period of 1 to 2 years, show much more efficacy when compared with results from treatment as usual studies. There is an increase in clinical response to VNS between 3 and 12 months, which is quite different from that seen with standard antidepressant treatment of MDD. Preclinically, VNS affects many of the same brain areas, neurotransmitters (serotonin, norepinephrine) and signal transduction mechanisms (brain-derived
\end{abstract}

Flavia R. Carreno

carrenof@uthscsa.edu

1 Department of Pharmacology, University of Texas Health Science Center at San Antonio, San Antonio, TX, USA

2 Center for Biomedical Neuroscience, University of Texas Health Science Center at San Antonio, San Antonio, TX, USA

3 South Texas Veterans Health Care System, San Antonio, TX, USA neurotrophic factor-tropomyosin receptor kinase B) as those found with traditional antidepressants. Nevertheless, the mechanisms by which VNS benefits patients nonresponsive to conventional antidepressants is unclear, with further research needed to clarify this.

Keywords TRD $\cdot$ VNS $\cdot$ BDNF-TrkB $\cdot$ Monoamines

Both worldwide and in the USA, major depressive disorder (MDD) is quite prevalent. In the USA, the lifetime prevalence is about $30 \%$, with the yearly prevalence being almost $9 \%$ [1]. Although antidepressant drugs are effective, their effect is mild to moderate, with selective serotonin reuptake inhibitors having effect sizes for acute response of 0.20 to 0.40 ; their ability to prevent relapses or recurrences is better, with effect sizes of 0.6 to 0.7 [2]. Another way to state this is that after 2 adequate trials with antidepressants, only about half the patients achieve remission, being defined as a $\geq 50 \%$ decrease in a severity rating scale score and a very low final score indicating minimal residual symptoms (see [3]). Remission is now viewed as the "gold standard" for treatment outcome as those with less residual symptoms after treatment for depression subsequently have less depressive symptoms, and better social functioning than those with more residual symptoms [4-6]. Just as importantly, up to $35 \%$ of patients with nonpsychotic MDD do not respond to 4 or more conventional treatments [7]. Such patients are considered to have treatment-resistant depression (TRD). Establishment of effective treatments for TRD would be very useful given the large number of patients who have it, their diminished quality of life and health $[8,9]$, the increased cost associated with it [9], and the overall chronic course of both MDD and TRD [10,11].

In view of such facts, much attention has been focused on trying to establish treatments that would at least have a 
modicum of effectiveness in such nonresponsive patients, either as standalone or adjunctive treatment. This review covers one such treatment, vagus nerve stimulation (VNS). It begins with a review of the evidence for its effectiveness and concludes with a review of effects it produces on brain function. A variety of neuromodulation techniques in addition to VNS have been tried in TRD, for example deep brain stimulation and repetitive transcranial magnetic stimulation. There are varying levels of evidence for their acute and long-term efficacy, as well as their safety (see [12]). Nevertheless, the Food and Drugs Administration approved VNS for treatment of TRD in 2005 and repetitive transcranial magnetic stimulation in 2008. The Food and Drugs Administration approved VNS as an adjunctive long-term treatment of chronic recurrent depression in patients 18 years of age or older who are experiencing a major depressive episode and have not had an adequate response to 4 or more adequate antidepressant treatments. It is important to note that the approval is for long-term treatment as the data reviewed below show efficacy to improve over months and even years but to be more limited over the first several months.

Interest in this topic has generated a number of review articles mentioned later. Most of these focus on clinical efficacy. Those that reviewed efficacy as well as potential mechanisms of action were published over a decade ago. This review covers both topics. Furthermore, it takes the position as discussed later, that the efficacy of VNS has to be compared with the response over time of patients with TRD not receiving VNS.

\section{The Vagus Nerve and VNS}

There is both an anatomical and functional rationale for stimulating the cervical vagus nerve to treat diseases of the brain. Since 1937, the vagus has been known to be mixed nerve with about $80 \%$ of its fibers carrying sensory afferent information to the brain and having about $20 \%$ efferent motor fibers [13]. Early evidence for the functionality of its afferent projection was the observation that its stimulation caused electroencephalography changes [14]. Its name comes from the Latin word for "wandering" owing to its having such a course along the esophagus and arteries to innervate numerous peripheral organs and structures (see [15]). Its visceromotor efferent component originates in the dorsal motor nucleus of the medulla, whereas the initial termination point of its afferent fibers in brain is primarily the nucleus tractus solitarius (NTS), also in the medulla. Neurotransmitters used by vagal afferents include peptides such as substance $\mathrm{P}$ and calcitonin generelated peptide and the excitatory amino acid transmitter Lglutamate.

The major outputs from the NTS can be characterized as 1) local projections to medullary motor nuclei; 2 ) projections to the midbrain including the locus coeruleus (LC), dorsal raphe nucleus (DRN) and other brainstem interneurons in the reticular formation and to the parabrachial nucleus (PBN); and 3) projections to forebrain sites such as the hypothalamus, amygdala, bed nucleus of the stria terminalis (BNST), and insular cortex (see [15-17]). And of course, there are projections from nuclei such as the LC and DRN to limbic forebrain areas. Thus, areas in brain long thought to be involved in behaviors relevant for depression are innervated, either directly or indirectly, from projections of afferent vagal fibers terminating in the NTS.

The VNS procedure involves implantation of a stimulation generator connected to bipolar electrodes that are placed around the left vagus nerve. The rationale for stimulating the left vagus nerve for TRD (or epilepsy) is that it innervates the $\mathrm{AV}$ node of the heart so as to have less of an effect on heart rate than the right vagus, which innervates the SA node. Stimulation of each vagus nerve produces effects on heart rate consistent with such innervation $[18,19]$. Further, the bipolar stimulating electrode is configured with the cathode at the proximal lead and the anode at the distal lead so as to direct action potential propagation to the central nervous system by creating an anodal block at the distal lead. This procedure is carried out as day surgery with local or general anesthesia. In the USA, such surgery is usually done by a neurosurgeon. The stimulation device is activated telemetrically by a wand connected to a hand-held computer. Stimulation parameters used include current $(\mathrm{mA})$, frequency $(\mathrm{Hz})$, pulse width $(\mu \mathrm{s})$, and duty cycle (the duration that stimulation is on or off). Such parameters reflect the "dose" of VNS. Stimulation is usually started with a low current, 0.25 to $0.75 \mathrm{~mA}$, which can be increased gradually. Frequencies on the order of 20 to $30 \mathrm{~Hz}$ are used clinically as frequencies of $50 \mathrm{~Hz}$ or higher can damage the vagus [20]. A pulse width of $250 \mu$ s and a duty cycle of stimulation for $30 \mathrm{~s}$ on/300 $\mathrm{s}$ off is often used.

\section{Clinical Studies}

As reported in Penry and Dean [21], VNS was used originally from 1988 to 1989 for inpatients with treatment-resistant epilepsy. In 2000, Elger et al. [22] were the first to note improvement of mood in patients with epilepsy that was independent of their seizure attenuation. Since 2005, almost 3000 clinical and preclinical papers dealing with VNS have been published, with many concerning its use in epilepsy or depression.

Given the importance of "dose" in producing a beneficial therapeutic response, it is somewhat surprising that the only prospective study examining dose of VNS for therapeutic outcome in TRD occurred quite recently, in 2013. Aaronson et al. [23] compared the response of patients with TRD to adjunctive VNS over 22 or 50 weeks and used 3 different "doses". The frequency and duty cycle were the same across the 3 
groups, whereas stimulation in the "low" group was $0.25 \mathrm{~mA}$ current with a $130 \mu$ s pulse width. For the "medium" group, the parameters were 0.5 to $1.0 \mathrm{~mA}$ and $250 \mu \mathrm{s}$, whereas it was 1.25 to $1.50 \mathrm{~mA}$ and $250 \mu \mathrm{s}$ for the "high" group. This study was a double-blind, randomized comparison of the effect of the different doses of VNS, but there was no control group (sham stimulation). The high dose was associated with less tolerability although $70 \%$ to $75 \%$ of patients in the high group reached their assigned dose. Similar efficacy was found in the 3 dose groups, that is, differences in outcome measures were not statistically significant. However, after 22 weeks of treatment, $10 \%$ to $20 \%$ of patients in the low group responded (depending on the scale used for efficacy) whereas $19 \%$ to $31 \%$ of patients did so in the high group. Consistent with data described below, behavioral improvement continued over time in that $25 \%$ of the patients who had not responded at 22 weeks did so at 50 weeks. Importantly, response was sustained up to 50 weeks in those who responded at 22 weeks, especially those receiving medium or high doses. Muller et al. [24] carried out a retrospective analysis of the effectiveness of 2 different doses of VNS in 2 groups of patients: 1) a lowstrength/high-frequency $(\leq 1.5 \mathrm{~mA}, 20 \mathrm{~Hz})$ group versus a high-strength/low-frequency $(>1.5 \mathrm{~mA}, 15 \mathrm{~Hz})$ group. Change in the Hamilton Rating Scale for Depression (HAMD) at 6-month intervals was the outcome measure. Better outcome was achieved with the low-strength/high-frequency group. Further prospective studies are clearly needed as there is evidence that VNS does have frequency-dependent effects in patients, as shown by results using functional magnetic resonance imaging (fMRI) [25].

In addition to original studies of the efficacy of VNS in TRD, there have been quite a few reviews of this topic [16, 26-34]. And, not surprisingly, the overall conclusion is that more research, particularly randomized control trials (RCTs), is needed before efficacy can be established conclusively. This is so, but our opinion is that the evidence for efficacy is quite substantial in that its effect has to be compared with the response over time of depressed patients resistant to treatment not receiving neurostimulation therapies such as VNS. And that response is quite modest. Dunner et al. [35] reported a prospective study of outcomes of 124 patients with TRD (15 of whom were bipolar) treated with standard care [i.e., treatment as usual (TAU)] over 2 years. After 12 months, the response rate was about $12 \%$ and it was $18.4 \%$ after 24 months. Remission rates were $3.6 \%$ and $7.8 \%$, respectively. And neither response nor remission was well-maintained over time with TAU. The emphasis on RCTs with VNS is likely based, in part, on the high placebo response rates in clinical trials of antidepressants. In such short-term trials, placebo response rates of almost $40 \%$ are usual [36]. Although placebo responses may be maintained for 12 weeks after stopping the clinical trial [37], maintaining recurrent depressives on placebo over 6 to 12 months is much less effective than maintaining them on drug [38]. Further, the current poor response of patients with TRD to TAU is noteworthy in light of the increase in placebo response rates since the 1980 s to 1990 s in nontreatment-resistant patients [39]. Thus, even though there is clearly a need for more long-term RCTs with VNS, the data reviewed below on patients with TRD should be considered in this context.

Based on results of earlier open trials of the short-term (10 weeks) efficacy of VNS in patients with TRD that showed response rates of $30 \%$ to $40 \%$ [40, 41], an RCT of 10 weeks of treatment with VNS versus sham stimulation was carried out [42]. On the primary HAMD response measure, VNS produced a response rate of $15.2 \%$ versus $10.0 \%$ in the sham group - this was not a significant difference. However, differences in response rates with a secondary outcome measure were significant. To date, this is the only RCT to employ sham stimulation as a comparison to VNS. Attention has been focused appropriately on this negative result and the fact that there is considerable variability in effectiveness among the studies [30]. And longerterm studies (e.g., 2 years) with VNS (reviewed below) can be influenced by the natural course of MDD. For example, Nahas et al. [43] followed the 59 patients in the original open 10-week study of Sackeim et al. [41] for 2 years. Response rate increased over time from $31 \%$ at 3 months to $42 \%$ to $44 \%$ at 12 to 24 months. Remission rates were about $25 \%$ after 1 to 2 years. And for those that responded at 3 months, $50 \%$ to $75 \%$ remained well at 12 to 24 months. Subsequently, with a larger cohort of 205 patients, of those who responded to VNS at 3 months, $76.7 \%$ maintained response at 24 months and for those who did not respond until 12 months, $65 \%$ were still responders at 24 months [44]. For patients with TRD, these response and remission rates are considerably higher than those achieved over a similar time with TAU [35] such that the natural course of treatment-resistant patients is different from those receiving treatment who are not resistant, where recovery rates of $60 \%$ to $85 \%$ are seen over 2 years $[45,46]$.

George et al. [47] attempted to put such results from this open trial into perspective by analyzing data from those treated with VNS for 1 year in open studies with results from comparable patients receiving TAU as reported by Dunner et al. [35]. Although there was not a priori randomization, the patient characteristics seem comparable with similar inclusion and exclusion criteria and demographics, with the studies being carried out over a similar time period. Also, 12 of the 13 sites for the TAU study participated in the VNS + TAU study with 9 additional sites only participating in the VNS + TAU study. Irrespective of the method of data analysis, the addition of VNS to TAU produced a greater reduction of depressive symptoms with the effect of VNS becoming greater the longer the treatment. Importantly, the effect of VNS was sustainable in that about $55 \%$ of those who responded in the VNS group 
were also responders at 12 months, whereas this percentage was only $11.5 \%$ in the TAU group.

Results similar to these from the USA were found in an open-label study enrolling patients with TRD from 6 European countries. Seventy patients were evaluated at 3 months with 60 at 12 months [48] and 49 at 24 months [49]. In the initial report [46], response rates of $37 \%$ and $53 \%$ were found after 3 and 12 months, respectively, whereas remission rates at these times were $13 \%$ and $33 \%$, respectively. In the follow-up 2-year study [47], 53\% fulfilled the response criterion and $39 \%$ did so for the remission criterion [49]. Furthermore, a reanalysis of this study was carried out by Christmas et al. [50] in which only patients who had failed 4 or more antidepressant trials were included. Here, a response rate of $35.7 \%$ was found after 12 months, somewhat lower than that found in the entire patient sample. Once again, initial response was reasonably well-maintained over time with almost $40 \%$ of those responding at 3 months still responding at 12 and 24 months. These studies, then, demonstrate response to VNS increasing over months with response being reasonably well maintained in these difficult-to-treat depressed patients.

Very recently, Aaronson et al. [51] published a 5-year follow-up to their previous study comparing the effects of VNS or TAU in patients with TRD carried out for 50 weeks. This study has the longest duration of treatment so far. Consistent with their earlier results, the group receiving adjunctive VNS had better clinical outcomes than the group receiving TAU, with those receiving VNS having a significantly higher 5-year cumulative response rate (67.6\% vs $40.9 \%)$ and a significantly higher remission rate (cumulative first-time remitters, $43.3 \%$ vs 25.7\%) than those getting TAU.

A recent review by Cimpianu et al. [28] systematically reviewed the evidence for the efficacy of VNS in TRD, as well as other psychiatric disorders. For the other disorders, there were scant data and no conclusions could be made. The interested reader is directed to this comprehensive review, particularly Table 1, which gives details about the 33 studies included in their quantitative analysis, with 24 using standard VNS for TRD [as opposed to transcutaneous VNS (see below)]. As mentioned, the evidence from these in general nonrandomized open studies carried out over long time periods indicates benefit from VNS. This view is supported by a meta-analysis of 6 outpatient multicenter clinical trials of VNS + TAU or TAU alone, all of which were sponsored by Cyberonics, Inc., the manufacturer of the stimulation device. This analysis found that adjunctive VNS therapy was associated with greater response and remission than TAU alone, at time periods from 12 to 96 weeks [26]. Retrospective observational studies also support this view such as one involving Medicare patients [52] where patients with TRD receiving VNS had lower yearly medical costs postimplantation than those receiving TAU, and reduced annual mortality rates.
Nevertheless, it is wise to be cautious in the absence of more RCTs of the effectiveness of VNS in TRD before reaching definitive conclusions. This is likely why some national guidelines recommend VNS for TRD, whereas others are more circumspect (see [28]). But patients with TRD need help today. Given that VNS is generally well tolerated [12, 16, 49], together with the negative consequences of TRD, it should definitely be considered in the armamentarium of treatments for TRD.

A relatively new development may aid in its use for TRD. It has now been established that in humans the auricular branch of the vagus nerve is close to the surface in the ear, particularly the middle-third and lower-third area of the medial surface of the auricle [53]. This observation led to the use of ear clips to stimulate the vagus at this site. Furthermore, other auricular areas do not receive vagal innervation, such as the superior scapha, and this permits sham stimulation to be carried out, as well as actual stimulation. This noninvasive stimulation of the vagus nerve is referred to as transcutaneous VNS (tVNS). Such stimulation produces sensory evoked potentials recorded from the scalp [54], which provides a rationale that this technique could be used to affect brain function. Now, several studies have shown that short-term VNS generates fMRI blood oxygen level-dependent signal activations in limbic and brainstem areas $[55,56]$. Furthermore, tVNS does cause changes in functional connectivity in brain in depressed patients, as measured using fMRI $[57,58]$. In these latter studies, stimulation was carried out for $30 \mathrm{~min}$ twice each day for 5 days each week over 4 weeks. So as with the invasive VNS procedure, there is both an anatomic and functional rationale for studying effects of auricular stimulation of the vagus nerve for different types of brain disorders.

Two studies have assessed the efficacy of tVNS in patients with MDD; there is no indication that these patients were treatment resistant. In the first study [59], 37 patients received stimulation for $15 \mathrm{~min}$ either once or twice a day, 5 days weekly, for a rather short time interval ( 2 weeks). Stimulation parameters were adjusted such that the intensity was just below the threshold of perception, that is, when the stimulus was just noticeable. Sham stimulation consisted of using similar electrodes but having no current applied. Effectiveness was evaluated using both the patient-rated Beck Depression Inventory (BDI) and the physician-completed HAMD. In comparison with the effect of sham stimulation, significant improvement in depressive symptomatology was found using the BDI. However, this was not so for the HAMD, possibly because the sham stimulation had a greater effect on the HAMD than the BDI. Rong et al. [60] studied a total of 160 patients, with 91 receiving only tVNS for 12 weeks, whereas 69 initially received sham stimulation for 4 weeks followed by 8 weeks of tVNS. In this study, sham stimulation did consist of actual stimulation but at a place on the ear not receiving a distribution from the vagus nerve. Stimulation occurred for 30 min twice each day for 5 days 
weekly, with the stimulation occurring at home. Stimulation intensity was adjusted to the highest point that the patients could tolerate. After 4 weeks of treatment, tVNS reduced HAMD scores significantly more than the reduction of those receiving sham stimulation and $27 \%$ of the patients were judged as responders after 4 weeks of tVNS, whereas no patient achieved response with sham stimulation. Further improvement continued at 8 and 12 weeks.

These preliminary results are promising, but obviously much more research needs to be carried out, not the least of which would be studies to determine the optimal frequency and duration to administer tVNS and whether it would be effective in patients with TRD.

\section{Preclinical Studies}

Before reviewing preclinical studies trying to ascertain the mechanism(s) of action of VNS, a brief overview of functional brain-imaging studies in humans treated with it is relevant as such research is helping to understand brain regions and circuits modulated by VNS either acutely or long term. A limitation of such studies, and indeed all clinical studies involving VNS, as mentioned later, is the fact that the study population remains on their drug regimen, which may involve multiple drugs, and can change during the course of the study. Blood cerebral flow, fMRI, and blood oxygen level-dependent fMRI reveal that acute VNS somewhat consistently causes changes in orbitofrontal, anterior cingulate, dorsolateral, prefrontal, and insular cortices, as well as the striatum, cerebellum, and brainstem [25, 61-64]. With regard to the chronic effects of VNS on brain regions, changes have been noted in the thalamus, prefrontal cortex (PFC), limbic system, hypothalamus, medulla, and cerebellum [62, 65-67], although there is some inconsistency with regard to the direction of the change caused by VNS.

As for tVNS in humans, Kraus et al. [56] studied healthy volunteers with electrical stimulation of the nerves in the left outer auditory canal. The brain areas activated by tVNS are consistent with those found with conventional VNS. In the same study, a control group receiving stimulation of the ear lobe instead of the left outer auditory canal showed no effect on limbic areas. Further, in patients with depression, the pattern of brain areas modulated by tVNS is also consistent with that obtained by conventional VNS [57, 68]. Recently, 4 weeks of VNS was found to alter the resting state functional connectivity between the right amygdala and left dorsolateral PFC as well as to enhance activation of the left insula, with such changes associated with improvement in depressive symptomatology $[58,69]$. A more recent study using fMRI aimed to optimize activation of brain areas by tVNS by comparing 4 stimulation locations in the ear: the inner tragus, inferoposterior wall of the ear canal, cymba conchae, and earlobe (sham). Only stimulation of the cymba conchae produced a significantly stronger activation in both the NTS and LC than did the sham stimulation [70]. There have not yet been any studies evaluating such activation in improving depressive symptomatology.

\section{Brain Areas Activated by VNS}

Studies with animals are important to elucidate the mechanisms by which VNS produces its effects without confounds of having other drugs on board (as is the case in the clinic), as well as to optimize therapeutic stimulation parameters to increase further the number of patients with TRD achieving response and remission following VNS treatment. Acute, as well as chronic (sustained), activation of brain circuits following VNS treatment have been studied by conventional immunohistochemistry for c-fos or $\Delta$ FosB respectively [71-73]. Both c-fos and $\Delta$ FosB are expressed following neuronal activation. On the one hand, c-fos expression peaks within 1 to $3 \mathrm{~h}$, being used as a marker of acute neuronal activation [74]. On the other hand, $\Delta$ FosB shows a time lag for its expression, but persists longer, so it is used as an indicator of sustained neuronal activation $[75,76]$.

Using nonanesthetized rats and stimulation parameters that do not cause cardiovascular activation, Cunningham et al. [71] reported that acute VNS treatment $(2 \mathrm{~h}$ ) induces c-fos expression in the NTS, paraventricular nucleus of the hypothalamus, PBN, BNST, and LC, but not in the cingulate cortex or DRN. VNS treatment for 2 weeks revealed significant increases in $\triangle$ FosB immunoreactivity in NTS, PBN, LC, peripeduncular nucleus, frontal cortex, cingulate cortex, hippocampus, basolateral amygdala, nucleus accumbens, BNST, and now the DRN [71, 72]. Results such as these show that VNS induces a rather complex pattern of activation of brain circuits implicated not exclusively in regulation of mood. That could perhaps expand the indications for the use of VNS (see [28]). In fact, some other clinical trials show some promise for the use of VNS for neurological diseases [77-79], ischemic stroke [80, 81], drug-seeking behavior [82], and trigeminal allodynia [83], amongst others.

Given the role of the vagus nerve in modulating the inflammatory reflex (see [84]), there has been a developing hypothesis that another putative mechanism by which VNS exerts its therapeutic effects in treating depression could be due to its role in decreasing proinflammatory cytokine synthesis $[85$, 86]. It is known that higher levels of proinflammatory cytokines are often measured in the serum or cerebrospinal fluid of depressed patients [87-89]. A small clinical trial [86] in patients with TRD showed that before VNS treatment such patients exhibited high levels of some proinflammatory cytokines. Three months following VNS treatment, an increased circulating level of anti-inflammatory cytokines was 
measured in the same patients, corroborating the hypothesis that VNS modulates the immune system [86]. More recently another clinical trial showed that VNS inhibited proinflammatory cytokine production [90]; however, this study was not done in patients with TRD, but rather in patients with rheumatoid arthritis that had the disease attenuated by VNS [90]. More studies evaluating the role of VNS in reducing inflammation in patients with TRD are needed.

\section{Effects on Biogenic Amine systems}

It has long been hypothesized that the mechanism of action of conventional antidepressants is associated with enhancement in neurotransmission within the serotonergic and or noradrenergic systems [91-95]. So, electrophysiological recordings from noradrenergic neurons in the LC or serotonergic neurons in the DRN were performed to study, selectively, the activation of these cell types following acute and chronic VNS treatments. These were followed by microdialysis studies to measure extracellular norepinephrine (NE) and serotonin (5-HT) in different brain areas upon acute and chronic VNS treatment. Consistent with the immunohistochemical studies, electrophysiological recordings revealed that VNS acutely increases spontaneous firing activity in noradrenergic neurons in the LC [96], but not in serotonergic neurons in the DRN. This result is consistent with a recent brain-imaging study using positron emission tomography and the selective $\alpha_{2}$ adrenergic receptor antagonist $\left[{ }^{11} \mathrm{C}\right]$ yohimbine in anesthetized minipigs that showed that acute VNS decreased $\alpha_{2}$ adrenergic receptor binding in limbic, thalamic, and cortical brain regions [97]. Only chronic (2 weeks) VNS treatment was able to enhance the firing rate of serotonergic neurons, and noradrenergic neurons in the LC remained activated by chronic treatment [96]. Lesion studies revealed that the chronic activation of serotonergic neurons in the DRN is downstream of VNS-induced activation of noradrenergic neurons in the LC. Moreover, the increased activity of the DRN neurons is mediated through activation of postsynaptic $\alpha_{1}$ adrenoreceptors [96].

As shown by microdialysis, VNS rapidly increases NE levels in the cortex, medial PFC, amygdala, and hippocampus [98-100]. This is consistent with the rapid activation of NE neurons in the LC, which is upstream of these terminal regions. VNS-induced increases in extracellular NE in the PFC and hippocampus was also reported following chronic treatment [101]. Chronic, but not acute, VNS treatment was also found to cause an increase in 5-HT levels in the DRN, but not in the hippocampus [101].

The effect of VNS on the dopaminergic system shows some peculiarity. Manta et al. [101] found that in spite of dopamine (DA) cells in the ventral tegmental area (VTA) decreasing their firing rate in response to 2 weeks of VNS, extracellular DA levels in the PFC and nucleus accumbens were increased. Perez et al. [102] found that 2 weeks of VNS treatment in freely moving rats did not affect the number of spontaneously active DA cells in the VTA, nor their firing rate or burst firing [102]. By contrast, in the same study Perez et al. found that in a model where VTA cells were spontaneously active, namely the MAM model of schizophrenia [103, 104], chronic VNS normalized the activity within the VTA cells [102]. This might imply some utility of VNS in the treatment of schizophrenia. To date, there has only been one study such as this, using tVNS [105] of the outer ear canal. In this 26week study, there was no significant improvement in schizophrenia symptomatology with tVNS versus sham tVNS.

\section{Preclinical Behavioral Effects of VNS}

The forced swim test (FST) has predictive validity for drugs or therapies that significantly improve depressive symptomatology in humans [106], whereas the NSFT is used to screen anxiolytic-like compounds or therapies [107]. Both acute and chronic VNS treatments decrease the time animals spend immobile in the FST, which is consistent with an antidepressant-like effect of VNS [71, 108, 109]. Chronic, but not acute treatment with VNS has an anxiolytic-like effect [108]. Lesioning either serotonergic or noradrenergic systems completely abolished the VNS-induced antidepressant and anxiolytic-like effects [108]. In the kainic acid rat model for temporal lobe epilepsy, Grimonprez et al. [110] found a decrease in saccharin preference, quite often associated with the human equivalent of anhedonia, a core symptom of depression. Such a decrease in saccharin preference was reversed upon VNS for 2 weeks. Such results support its clinical efficacy in patients with anhedonia.

\section{Effect on Neurogenesis and Neurotrophins}

The neurogenesis theory of depression is based on the findings that there is a stress-induced decrease in adult neurogenesis in the hippocampus, and that treatment with antidepressants reverse such deficit in neurogenesis [111-113]. Biggio et al. [114] examined the effects of acute $(3 \mathrm{~h})$ and chronic (1 month) VNS upon cell proliferation and found that the number of BrdU-positive cells in the dentate gyrus was significantly increased $24 \mathrm{~h}$ and 3 weeks after treatment. Another study by Revesz et al. [115] also showed that acute $(48 \mathrm{~h})$ VNS increased cell proliferation in the hippocampus. Another studied looking at the effects of chronic VNS on hippocampal neurogenesis in an animal model of depression, namely bulbectomy, revealed that the bulbectomy-induced decrease in neuronally differentiated BrdU-positive cells 
within the dentate gyrus was prevented by VNS but not by sham stimulation [116].

Unlike positive results with acute VNS, acute treatment with traditional ADs does not increase neurogenesis; however, acute ECT, also used for TRD, does [117]. Antidepressants typically take 2 weeks to promote an enhancement in proliferation, but the outcome is rather variable [118-121]. Doublecortin (DCX) is a microtubule-associated protein used as a marker for neurogenesis, as it is expressed by neuronal precursor, as well as immature neurons, for about 2 weeks. Once they differentiate into mature neurons, they no longer express DCX $[122,123]$. Both, acute and chronic VNS were associated with significantly higher expression of DCX in the dentate gyrus for up to 3 weeks after discontinuation of treatment [114]. Similar results were found after chronic treatment with fluoxetine [124]. This topic has been reviewed recently [125].

As with the neurogenesis theory, the neurotrophic theory of depression is based on findings that neurotrophic factor expression in brain circuits associated with mood regulation is inversely proportional to the effects of stress and antidepressants. One such neurotrophic factor widely studied in this framework is brain-derived neurotrophic factor (BDNF), along with its receptor, namely tropomyosin receptor kinase B (TrkB). From a functional perspective, the neurotrophic hypothesis is linked to the neurogenesis hypothesis as the increase in expression of neurotrophins with antidepressant treatment may block or reverse the neuronal loss associated with depression [126]. Chronic treatment (21 days) with classical antidepressants belonging to different classes increases expression of mRNAs for BDNF and trkB in the hippocampus (CA1, CA3, and dentate gyrus) [127, 128]. Acute (3 h) VNS treatment increases mRNA for BDNF in the hippocampus and cortex [98].

As reviewed by Shah et al. [33], drawing conclusions based on changes in mRNA for BDNF or protein levels can be complicated owing to factors such as translation, proteolytic cleavage, and release that are not accounted for. Hence, analyzing activation of its receptor, TrkB, may provide additional clues about the effect of antidepressant treatment on BDNF function. We have shown that both acute $(2 \mathrm{~h})$ and chronic (14 days) VNS activates the TrkB receptor, as shown by its causing phosphorylation at 3 tyrosine residues (Y705, Y816, and Y515) on TrkB [129]. Intraventricular pretreatment with a scavenger compound for TrkB, namely TrkB-Fc, which is a homodimer containing the extracellular ligand-binding domains of TrkB linked to human IgG1, prevented the acute VNS-induced increase in TrkB phosphorylation [130]. This suggests that VNS-induced activation of TrkB requires ligand to bind to it [130]. In contrast to the effects of VNS on the phosphorylation of TrkB, acute and chronic antidepressant treatments only cause phosphorylation at Y705 and Y816 but not at Y $515[129,131,132]$. Y705 is the autophosphorylation site, whereas Y816 and Y515 are linked with the phospholipase C gamma 1 (PLC $\gamma 1$ ) and MAPK/ PI3K signaling pathways, respectively. Consistent with the phosphorylation of these tyrosine residues, acute VNS, as well as antidepressants, cause phosphorylation of PLC $\gamma 1$ [133]; however, this is not maintained with chronic treatments. Only acute and chronic VNS, but not antidepressants, cause phosphorylation of ERK1/2 and Akt that are downstream of Y515 [129, 133]. A recent study by Shah et al. [130] show that intracerebroventricular pretreatment with K252a, an inhibitor of receptor tyrosine kinases, blocks the anxiolytic-like effect of VNS ( 2 weeks) in the NSFT. Intracerebroventricular pretreatment with K252a also blocked the acute effects of VNS in the FST. Such results indicate that the activation of TrkB by BDNF may be necessary for these behavioral effects of VNS.

As is so clinically, effects of noninvasive tVNS have not yet been intensively studied preclinically. In the Zucker diabetic fatty rat (fa/fa), in addition to the metabolic disarrangements a depressive-like phenotype is observed as seen by an increase in time spent immobile in the FST. Chronic (4 weeks), $30 \mathrm{~min}$ daily tVNS ameliorated the depressive-like phenotype [134]. In this study, 2 opposite magnetic electrodes were placed in the right auricular concha regions, inside and out for the cathode and anode, respectively. Finally, although not tVNS, it has been found that electroacunpunture of the auricular concha region, which is densely innervated by nerve endings from the vagus nerve, abolished the behavioral and neurochemical deficits caused by the unpredictable chronic mild stress paradigm [135]. Future studies regarding tVNS or electroacunpunture of the auricular concha regions in rodents need to be performed to evaluate which brain areas are being activated by such paradigms and the effects they produce in such areas, as has now been done for VNS.

In conclusion, VNS has been found to affect many of the same brain areas, neurotransmitters, and signal transduction mechanisms as altered by conventional antidepressants. More often than not, similarities have been found between preclinical effects of VNS and traditional antidepressants, although there are some exceptions $[117,133]$. In spite of this, it is not clear what VNS is doing to allow it to be effective in patients when traditional antidepressants are not.

Further, the time course of clinical response to VNS with considerable improvement being noted between 3 to 12 months is quite different from the time course of druginduced improvement of MDD, where optimal improvement often occurs in 8 to 12 weeks. This difference in the time course of clinical response might indicate some fundamental difference in the mechanisms of action of VNS, that is, indicating time-dependent effects not caused by traditional antidepressants. Another complexity in understanding effects of VNS is the absence of validated, widely used models for TRD, although some have been proposed [136-140]. Also, 
animal studies are not carried out on the time scale where VNS is effective. Finally, animal studies have used VNS in the absence of other drugs on board. Yet this is not how VNS is used clinically where, as mentioned, it is added to TAU, which might involve multiple drugs and changing them. Whether there is some interaction between VNS and the effects of such drugs, in particular antidepressants, needs further study.

Acknowledgements We thank William R. Buras, formerly an employee of Cyberonics, Inc., the manufacturer of the VNS device, who is currently at Tietronix, Inc., Houston, TX, for reviewing and giving insightful suggestions on an earlier version of this manuscript. Dr. Frazer has not had involvements with Cyberonics, Inc. for over 5 years. He had previously been on one of their advisory boards and has received a grant from Cyberonics for some of his preclinical studies involving VNS. Dr. Carreno has no financial conflicts to disclose.

Required Author Forms Disclosure forms provided by the authors are available with the online version of this article.

\section{References}

1. Kessler, R.C., S. Avenevoli, K.A. McLaughlin, J.G. Green, M.D. Lakoma, et al., Lifetime co-morbidity of DSM-IV disorders in the US National Comorbidity Survey Replication Adolescent Supplement (NCS-A). Psychol Med, 2012. 42(9): p. 1997-2010.

2. Leucht, S., S. Hierl, W. Kissling, M. Dold, and J.M. Davis, Putting the efficacy of psychiatric and general medicine medication into perspective: review of meta-analyses. Br J Psychiatry, 2012. 200(2): p. 97-106.

3. Rush, A.J., Limitations in efficacy of antidepressant monotherapy. J Clin Psychiatry, 2007. 68 Suppl 10: p. 8-10.

4. Kennedy, N. and K. Foy, The impact of residual symptoms on outcome of major depression. Curr Psychiatry Rep, 2005. 7(6): p. 441-446.

5. Kennedy, N. and E.S. Paykel, Residual symptoms at remission from depression: impact on long-term outcome. J Affect Disord, 2004. 80(2-3): p. 135-144.

6. Pintor, L., C. Gasto, V. Navarro, X. Torres, and L. Fananas, Relapse of major depression after complete and partial remission during a 2-year follow-up. J Affect Disord, 2003. 73(3): p. 237244.

7. Rush, A.J., H.C. Kraemer, H.A. Sackeim, M. Fava, M.H. Trivedi, et al., Report by the ACNP Task Force on response and remission in major depressive disorder. Neuropsychopharmacology, 2006. 31(9): p. 1841-1853.

8. Fekadu, A., S.C. Wooderson, K. Markopoulo, C. Donaldson, A. Papadopoulos, et al., What happens to patients with treatmentresistant depression? A systematic review of medium to long term outcome studies. J Affect Disord, 2009. 116(1-2): p. 4-11.

9. Mrazek, D.A., J.C. Hornberger, C.A. Altar, and I. Degtiar, A review of the clinical, economic, and societal burden of treatmentresistant depression: 1996-2013. Psychiatr Serv, 2014. 65(8): p. 977-987.

10. Prudic, J., M. Olfson, S.C. Marcus, R.B. Fuller, and H.A. Sackeim, Effectiveness of electroconvulsive therapy in community settings. Biol Psychiatry, 2004. 55(3): p. 301-312.

11. Stegenga, B.T., M.H. Kamphuis, M. King, I. Nazareth, and M.I. Geerlings, The natural course and outcome of major depressive disorder in primary care: the PREDICT-NL study. Soc Psychiatry Psychiatr Epidemiol, 2012. 47(1): p. 87-95.
12. Bewernick, B. and T.E. Schlaepfer, Update on Neuromodulation for Treatment-Resistant Depression. F1000Res, 2015. 4.

13. Foley, J.O. and F.S. DuBois, Quantitative studies of the vagus nerve in the cat. I. The ratio of sensory to motor fibers. J. Comp. Neurol., 1937. 67: p. 49-67.

14. Bailey, P. and F. Bremer, A sensory cortical representation of the vagus nerve. J Neurophysiol, 1938. 1:p. 405-412.

15. Berthoud, H.R. and W.L. Neuhuber, Functional and chemical anatomy of the afferent vagal system. Auton Neurosci, 2000. 85(1-3): p. 1-17.

16. George, M.S. and G. Aston-Jones, Noninvasive techniques for probing neurocircuitry and treating illness: vagus nerve stimulation (VNS), transcranial magnetic stimulation (TMS) and transcranial direct current stimulation (tDCS). Neuropsychopharmacology, 2010. 35(1): p. 301-316.

17. Yuan, H. and S.D. Silberstein, Vagus Nerve and Vagus Nerve Stimulation, a Comprehensive Review: Part I. Headache, 2016. 56(1): p. 71-78.

18. Randall, W.C. and J.L. Ardell, Selective parasympathectomy of automatic and conductile tissues of the canine heart. Am J Physiol, 1985. 248(1 Pt 2): p. H61-H68.

19. Woodbury, D.M. and J.W. Woodbury, Effects of vagal stimulation on experimentally induced seizures in rats. Epilepsia, 1990. 31 Suppl 2: p. S7-S19.

20. Agnew, W.F. and D.B. McCreery, Considerations for safety with chronically implanted nerve electrodes. Epilepsia, 1990. 31 Suppl 2: p. S27-S32.

21. Penry, J.K. and J.C. Dean, Prevention of intractable partial seizures by intermittent vagal stimulation in humans: preliminary results. Epilepsia, 1990. 31 Suppl 2: p. S40-S43.

22. Elger, G., C. Hoppe, P. Falkai, A.J. Rush, and C.E. Elger, Vagus nerve stimulation is associated with mood improvements in epilepsy patients. Epilepsy Res, 2000. 42(2-3): p. 203-210.

23. Aaronson, S.T., L.L. Carpenter, C.R. Conway, F.W. Reimherr, S.H. Lisanby, et al., Vagus nerve stimulation therapy randomized to different amounts of electrical charge for treatment-resistant depression: acute and chronic effects. Brain Stimul, 2013. 6(4): p. 631-640.

24. Muller, H.H., J. Kornhuber, J.M. Maler, and W. Sperling, The effects of stimulation parameters on clinical outcomes in patients with vagus nerve stimulation implants with major depression. $\mathbf{J}$ ECT, 2013. 29(3): p. e40-e42.

25. Lomarev, M., S. Denslow, Z. Nahas, J.H. Chae, M.S. George, et al., Vagus nerve stimulation (VNS) synchronized BOLD fMRI suggests that VNS in depressed adults has frequency/dose dependent effects. J Psychiatr Res, 2002. 36(4): p. 219-227.

26. Berry, S.M., K. Broglio, M. Bunker, A. Jayewardene, B. Olin, et al., A patient-level meta-analysis of studies evaluating vagus nerve stimulation therapy for treatment-resistant depression. Med Devices (Auckl), 2013. 6: p. 17-35.

27. Blumberger, D.M., J.H. Hsu, and Z.J. Daskalakis, A Review of Brain Stimulation Treatments for Late-Life Depression. Curr Treat Options Psychiatry, 2015. 2(4): p. 413-421.

28. Cimpianu, C.L., W. Strube, P. Falkai, U. Palm, and A. Hasan, Vagus nerve stimulation in psychiatry: a systematic review of the available evidence. J Neural Transm (Vienna), 2017; 124: 145-158.

29. Groves, D.A. and V.J. Brown, Vagal nerve stimulation: a review of its applications and potential mechanisms that mediate its clinical effects. Neurosci Biobehav Rev, 2005. 29(3): p. 493-500.

30. Martin, J.L. and E. Martin-Sanchez, Systematic review and metaanalysis of vagus nerve stimulation in the treatment of depression: variable results based on study designs. Eur Psychiatry, 2012. 27(3): p. 147-155.

31. Nemeroff, C.B., H.S. Mayberg, S.E. Krahl, J. McNamara, A. Frazer, et al., VNS therapy in treatmentresistant depression: 
clinical evidence and putative neurobiological mechanisms. Neuropsychopharmacology, 2006. 31(7): p. 1345-1355.

32. Rush, A.J. and S.E. Siefert, Clinical issues in considering vagus nerve stimulation for treatment-resistant depression. Exp Neurol, 2009. 219(1): p. 36-43.

33. Shah, A., F.R. Carreno, and A. Frazer, Therapeutic modalities for treatment resistant depression: focus on vagal nerve stimulation and ketamine. Clin Psychopharmacol Neurosci, 2014. 12(2): p. 83-93.

34. Beekwilder, J.P. and T. Beems, Overview of the clinical applications of vagus nerve stimulation. J Clin Neurophysiol, 2010. 27(2): p. 130-138.

35. Dunner, D.L., A.J. Rush, J.M. Russell, M. Burke, S. Woodard, et al., Prospective, long-term, multicenter study of the naturalistic outcomes of patients with treatment-resistant depression. J Clin Psychiatry, 2006. 67(5): p. 688-695.

36. Freeman, M.P., D. Mischoulon, E. Tedeschini, T. Goodness, L.S. Cohen, et al., Complementary and alternative medicine for major depressive disorder: a meta-analysis of patient characteristics, placeboresponse rates, and treatment outcomes relative to standard antidepressants. J Clin Psychiatry, 2010. 71(6): p. 682-688.

37. Khan, A., N. Redding, and W.A. Brown, The persistence of the placebo response in antidepressant clinical trials. J Psychiatr Res, 2008. 42(10): p. 791-796.

38. Borges, S., Y.F. Chen, T.P. Laughren, R. Temple, H.D. Patel, et al., Review of maintenance trials for major depressive disorder: a 25year perspective from the US Food and Drug Administration. J Clin Psychiatry, 2014. 75(3): p. 205-214.

39. Undurraga, J. and R.J. Baldessarini, Randomized, placebo-controlled trials of antidepressants for acute major depression: thirtyyear meta-analytic review. Neuropsychopharmacology, 2012. 37(4): p. 851-864.

40. Rush, A.J., M.S. George, H.A. Sackeim, L.B. Marangell, M.M. Husain, et al., Vagus nerve stimulation (VNS) for treatment-resistant depressions: a multicenter study. Biol Psychiatry, 2000. 47(4): p. 276-286.

41. Sackeim, H.A., A.J. Rush, M.S. George, L.B. Marangell, M.M. Husain, et al., Vagus nerve stimulation (VNS) for treatment-resistant depression: efficacy, side effects, and predictors of outcome. Neuropsychopharmacology, 2001. 25(5): p. 713-728.

42. Rush, A.J., L.B. Marangell, H.A. Sackeim, M.S. George, S.K. Brannan, et al., Vagus nerve stimulation for treatment-resistant depression: a randomized, controlled acute phase trial. Biol Psychiatry, 2005. 58(5): p. 347-354.

43. Nahas, Z., L.B. Marangell, M.M. Husain, A.J. Rush, H.A. Sackeim, et al., Two-year outcome of vagus nerve stimulation (VNS) for treatment of major depressive episodes. J Clin Psychiatry, 2005. 66(9): p. 1097-1104.

44. Sackeim, H.A., S.K. Brannan, A.J. Rush, M.S. George, L.B. Marangell, et al., Durability of antidepressant response to vagus nerve stimulation (VNS). Int J Neuropsychopharmacol, 2007. 10(6): p. 817-826.

45. Coryell, W., H.S. Akiskal, A.C. Leon, G. Winokur, J.D. Maser, et al., The time course of nonchronic major depressive disorder. Uniformity across episodes and samples. National Institute of Mental Health Collaborative Program on the Psychobiology of Depression-Clinical Studies. Arch Gen Psychiatry, 1994. 51(5): p. 405-410.

46. Eaton, W.W., H. Shao, G. Nestadt, H.B. Lee, O.J. Bienvenu, et al., Population-based study of first onset and chronicity in major depressive disorder. Arch Gen Psychiatry, 2008. 65(5): p. 513-520.

47. George, M.S., A.J. Rush, L.B. Marangell, H.A. Sackeim, S.K. Brannan, et al., A one-year comparison of vagus nerve stimulation with treatment as usual for treatment-resistant depression. Biol Psychiatry, 2005. 58(5): p. 364-373.
48. Schlaepfer, T.E., C. Frick, A. Zobel, W. Maier, I. Heuser, et al., Vagus nerve stimulation for depression: efficacy and safety in a European study. Psychol Med, 2008. 38(5): p. 651-661.

49. Bajbouj, M., A. Merkl, T.E. Schlaepfer, C. Frick, A. Zobel, et al., Two-year outcome of vagus nerve stimulation in treatment-resistant depression. J Clin Psychopharmacol, 2010. 30(3): p. 273-281.

50. Christmas, D., J.D. Steele, S. Tolomeo, M.S. Eljamel, and K. Matthews, Vagus nerve stimulation for chronic major depressive disorder: 12-month outcomes in highly treatment-refractory patients. J Affect Disord, 2013. 150(3): p. 1221-1225.

51. Aaronson, S.T., P. Sears, F. Ruvuna, M. Bunker, C.R. Conway, et al., A 5-Year Observational Study of Patients With TreatmentResistant Depression Treated With Vagus Nerve Stimulation or Treatment as Usual: Comparison of Response, Remission, and Suicidality. Am J Psychiatry, 2017 Mar 31 [Epub ahead of print].

52. Feldman, R.L., D.L. Dunner, J.S. Muller, and D.A. Stone, Medicare patient experience with vagus nerve stimulation for treatment-resistant depression. J Med Econ, 2013. 16(1): p. 62-74.

53. Peuker, E.T. and T.J. Filler, The nerve supply of the human auricle. Clin Anat, 2002. 15(1): p. 35-37.

54. Fallgatter, A.J., B. Neuhauser, M.J. Herrmann, A.C. Ehlis, A. Wagener, et al., Far field potentials from the brain stem after transcutaneous vagus nerve stimulation. J Neural Transm (Vienna), 2003. 110(12): p. 1437-1443.

55. Dietrich, S., J. Smith, C. Scherzinger, K. Hofmann-Preiss, T. Freitag, et al., [A novel transcutaneous vagus nerve stimulation leads to brainstem and cerebral activations measured by functional MRI]. Biomed Tech (Berl), 2008. 53(3): p. 104-111.

56. Kraus, T., K. Hosl, O. Kiess, A. Schanze, J. Kornhuber, et al., BOLD fMRI deactivation of limbic and temporal brain structures and mood enhancing effect by transcutaneous vagus nerve stimulation. J Neural Transm (Vienna), 2007. 114(11): p. 1485-1493.

57. Fang, J., P. Rong, Y. Hong, Y. Fan, J. Liu, et al., Transcutaneous Vagus Nerve Stimulation Modulates Default Mode Network in Major Depressive Disorder. Biol Psychiatry, 2016. 79(4): p. 266273.

58. Liu, J., J. Fang, Z. Wang, P. Rong, Y. Hong, et al., Transcutaneous vagus nerve stimulation modulates amygdala functional connectivity in patients with depression. J Affect Disord, 2016. 205: p. 319-326.

59. Hein, E., M. Nowak, O. Kiess, T. Biermann, K. Bayerlein, et al., Auricular transcutaneous electrical nerve stimulation in depressed patients: a randomized controlled pilot study. J Neural Transm (Vienna), 2013. 120(5): p. 821-827.

60. Rong, P., J. Liu, L. Wang, R. Liu, J. Fang, et al., Effect of transcutaneous auricular vagus nerve stimulation on major depressive disorder: A nonrandomized controlled pilot study. J Affect Disord, 2016. 195: p. 172-179.

61. Bohning, D.E., M.P. Lomarev, S. Denslow, Z. Nahas, A. Shastri, et al., Feasibility of vagus nerve stimulation-synchronized blood oxygenation level-dependent functional MRI. Invest Radiol, 2001. 36(8): p. 470-479.

62. Conway, C.R., J.T. Chibnall, M.A. Gebara, J.L. Price, A.Z. Snyder, et al., Association of cerebral metabolic activity changes with vagus nerve stimulation antidepressant response in treatment-resistant depression. Brain Stimul, 2013. 6(5): p. 788-797.

63. Mu, Q., D.E. Bohning, Z. Nahas, J. Walker, B. Anderson, et al., Acute vagus nerve stimulation using different pulse widths produces varying brain effects. Biol Psychiatry, 2004. 55(8): p. 816825.

64. Nahas, Z., C. Teneback, J.H. Chae, Q. Mu, C. Molnar, et al., Serial vagus nerve stimulation functional MRI in treatment-resistant depression. Neuropsychopharmacology, 2007. 32(8): p. 1649-1660.

65. Chae, J.H., Z. Nahas, M. Lomarev, S. Denslow, J.P. Lorberbaum, et al., A review of functional neuroimaging studies of vagus nerve stimulation (VNS). J Psychiatr Res, 2003. 37(6): p. 443-455. 
66. Conway, C.R., Y.I. Sheline, J.T. Chibnall, R.D. Bucholz, J.L. Price, et al., Brain blood-flow change with acute vagus nerve stimulation in treatment-refractory major depressive disorder. Brain Stimul, 2012. 5(2): p. 163-171.

67. Kosel, M., H. Brockmann, C. Frick, A. Zobel, and T.E. Schlaepfer, Chronic vagus nerve stimulation for treatment-resistant depression increases regional cerebral blood flow in the dorsolateral prefrontal cortex. Psychiatry Res, 2011. 191(3): p. 153159.

68. Frangos, E., J. Ellrich, and B.R. Komisaruk, Non-invasive Access to the Vagus Nerve Central Projections via Electrical Stimulation of the External Ear: fMRI Evidence in Humans. Brain Stimul, 2015. 8(3): p. 624-636.

69. Fang, J., N. Egorova, P. Rong, J. Liu, Y. Hong, et al., Early cortical biomarkers of longitudinal transcutaneous vagus nerve stimulation treatment success in depression. Neuroimage Clin, 2017. 14: p. 105-111.

70. Yakunina, N., S.S. Kim, and E.C. Nam, Optimization of Transcutaneous Vagus Nerve Stimulation Using Functional MRI. Neuromodulation, 2017. 20(3): p. 290-300.

71. Cunningham, J.T., S.W. Mifflin, G.G. Gould, and A. Frazer, Induction of c-Fos and DeltaFosB immunoreactivity in rat brain by Vagal nerve stimulation. Neuropsychopharmacology, 2008. 33(8): p. 1884-1895.

72. Furmaga, H., M. Sadhu, and A. Frazer, Comparison of DeltaFosB immunoreactivity induced by vagal nerve stimulation with that caused by pharmacologically diverse antidepressants. J Pharmacol Exp Ther, 2012. 341(2): p. 317-325.

73. Naritoku, D.K., W.J. Terry, and R.H. Helfert, Regional induction of fos immunoreactivity in the brain by anticonvulsant stimulation of the vagus nerve. Epilepsy Res, 1995. 22(1): p. 53-62.

74. Kovacs, K.J., c-Fos as a transcription factor: a stressful (re)view from a functional map. Neurochem Int, 1998. 33(4): p. 287-297.

75. Chen, J., M.B. Kelz, B.T. Hope, Y. Nakabeppu, and E.J. Nestler, Chronic Fos-related antigens: stable variants of deltaFosB induced in brain by chronic treatments. J Neurosci, 1997. 17(13): p. 49334941.

76. Nestler, E.J., Molecular mechanisms of drug addiction. Neuropharmacology, 2004. 47 Suppl 1: p. 24-32.

77. Engineer, C.T., N.D. Engineer, J.R. Riley, J.D. Seale, and M.P. Kilgard, Pairing Speech Sounds With Vagus Nerve Stimulation Drives Stimulus-specific Cortical Plasticity. Brain Stimul, 2015. 8(3): p. 637-644.

78. Hays, S.A., R.L. Rennaker, and M.P. Kilgard, Targeting plasticity with vagus nerve stimulation to treat neurological disease. Prog Brain Res, 2013. 207: p. 275-299.

79. Shetake, J.A., N.D. Engineer, W.A. Vrana, J.T. Wolf, and M.P. Kilgard, Pairing tone trains with vagus nerve stimulation induces temporal plasticity in auditory cortex. Exp Neurol, 2012. 233(1): p. 342-349.

80. Khodaparast, N., S.A. Hays, A.M. Sloan, T. Fayyaz, D.R. Hulsey, et al., Vagus nerve stimulation delivered during motor rehabilitation improves recovery in a rat model of stroke. Neurorehabil Neural Repair, 2014. 28(7): p. 698-706.

81. Khodaparast, N., S.A. Hays, A.M. Sloan, D.R. Hulsey, A. Ruiz, et al., Vagus nerve stimulation during rehabilitative training improves forelimb strength following ischemic stroke. Neurobiol Dis, 2013. 60: p. 80-88.

82. Childs, J.E., J. DeLeon, E. Nickel, and S. Kroener, Vagus nerve stimulation reduces cocaine seeking and alters plasticity in the extinction network. Learn Mem, 2017. 24(1): p. 35-42.

83. Oshinsky, M.L., A.L. Murphy, H. Hekierski, Jr., M. Cooper, and B.J. Simon, Noninvasive vagus nerve stimulation as treatment for trigeminal allodynia. Pain, 2014. 155(5): p. 1037-1042.
84. Pavlov, V.A. and K.J. Tracey, The vagus nerve and the inflammatory reflex-linking immunity and metabolism. Nat Rev Endocrinol, 2012. 8(12): p. 743-754.

85. Das, U.N., Is depression a low-grade systemic inflammatory condition? Am J Clin Nutr, 2007. 85(6): p. 1665-1666.

86. Corcoran, C., T.J. Connor, V. O'Keane, and M.R. Garland, The effects of vagus nerve stimulation on proand anti-inflammatory cytokines in humans: a preliminary report. Neuroimmunomodulation, 2005. 12(5): p. 307-309.

87. Levine, J., Y. Barak, K.N. Chengappa, A. Rapoport, M. Rebey, et al., Cerebrospinal cytokine levels in patients with acute depression. Neuropsychobiology, 1999. 40(4): p. 171-176.

88. Suarez, E.C., R.R. Krishnan, and J.G. Lewis, The relation of severity of depressive symptoms to monocyte-associated proinflammatory cytokines and chemokines in apparently healthy men. Psychosom Med, 2003. 65(3): p. 362-368.

89. Lindqvist, D., S. Janelidze, P. Hagell, S. Erhardt, M. Samuelsson, et al., Interleukin-6 is elevated in the cerebrospinal fluid of suicide attempters and related to symptom severity. Biol Psychiatry, 2009. 66(3): p. 287-292.

90. Koopman, F.A., S.S. Chavan, S. Miljko, S. Grazio, S. Sokolovic, et al., Vagus nerve stimulation inhibits cytokine production and attenuates disease severity in rheumatoid arthritis. Proc Natl Acad Sci U S A, 2016. 113(29): p. 8284-8289.

91. Delgado, P.L., D.S. Charney, L.H. Price, G.K. Aghajanian, H. Landis, et al., Serotonin function and the mechanism of antidepressant action. Reversal of antidepressant-induced remission by rapid depletion of plasma tryptophan. Arch Gen Psychiatry, 1990. 47(5): p. 411-418.

92. Gyermek, L., The pharmacology of imipramine and related antidepressants. Int Rev Neurobiol, 1966. 9: p. 95-143.

93. Heninger, G.R., P.L. Delgado, and D.S. Charney, The revised monoamine theory of depression: a modulatory role for monoamines, based on new findings from monoamine depletion experiments in humans. Pharmacopsychiatry, 1996. 29(1): p. 2-11.

94. Prange, A.J., Jr., The Pharmacology and Biochemistry of Depression. Dis Nerv Syst, 1964. 25: p. 217-221.

95. Schildkraut, J.J., The catecholamine hypothesis of affective disorders: a review of supporting evidence. Am J Psychiatry, 1965. 122(5): p. 509-522.

96. Manta, S., J. Dong, G. Debonnel, and P. Blier, Enhancement of the function of rat serotonin and norepinephrine neurons by sustained vagus nerve stimulation. J Psychiatry Neurosci, 2009. 34(4): p. 272-280.

97. Landau, A.M., S. Dyve, S. Jakobsen, A.K. Alstrup, A. Gjedde, et al., Acute Vagal Nerve Stimulation Lowers alpha2 Adrenoceptor Availability: Possible Mechanism of Therapeutic Action. Brain Stimul, 2015. 8(4): p. 702-707.

98. Follesa, P., F. Biggio, G. Gorini, S. Caria, G. Talani, et al., Vagus nerve stimulation increases norepinephrine concentration and the gene expression of BDNF and bFGF in the rat brain. Brain Res, 2007. 1179: p. 28-34.

99. Hassert, D.L., T. Miyashita, and C.L. Williams, The effects of peripheral vagal nerve stimulation at a memory-modulating intensity on norepinephrine output in the basolateral amygdala. Behav Neurosci, 2004. 118(1): p. 79-88.

100. Roosevelt, R.W., D.C. Smith, R.W. Clough, R.A. Jensen, and R.A. Browning, Increased extracellular concentrations of norepinephrine in cortex and hippocampus following vagus nerve stimulation in the rat. Brain Res, 2006. 1119(1): p. 124-132.

101. Manta, S., M. El Mansari, G. Debonnel, and P. Blier, Electrophysiological and neurochemical effects of long-term vagus nerve stimulation on the rat monoaminergic systems. Int $\mathrm{J}$ Neuropsychopharmacol, 2013. 16(2): p. 459-470. 
102. Perez, S.M., F.R. Carreno, A. Frazer, and D.J. Lodge, Vagal nerve stimulation reverses aberrant dopamine system function in the methylazoxymethanol acetate rodent model of schizophrenia. $\mathrm{J}$ Neurosci, 2014. 34(28): p. 9261-9267.

103. Lodge, D.J., The MAM rodent model of schizophrenia. Curr Protoc Neurosci, 2013. Chapter 9: p. Unit9 43.

104. Moore, H., J.D. Jentsch, M. Ghajarnia, M.A. Geyer, and A.A. Grace, A neurobehavioral systems analysis of adult rats exposed to methylazoxymethanol acetate on E17: implications for the neuropathology of schizophrenia. Biol Psychiatry, 2006. 60(3): p. 253-264.

105. Hasan, A., C. Wolff-Menzler, S. Pfeiffer, P. Falkai, E. Weidinger, et al., Transcutaneous noninvasive vagus nerve stimulation (tVNS) in the treatment of schizophrenia: a bicentric randomized controlled pilot study. Eur Arch Psychiatry Clin Neurosci, 2015. 265(7): p. 589-600.

106. Porsolt, R.D., A. Bertin, and M. Jalfre, Behavioral despair in mice: a primary screening test for antidepressants. Arch Int Pharmacodyn Ther, 1977. 229(2): p. 327-336.

107. Bodnoff, S.R., B. Suranyi-Cadotte, D.H. Aitken, R. Quirion, and M.J. Meaney, The effects of chronic antidepressant treatment in an animal model of anxiety. Psychopharmacology (Berl), 1988. 95(3): p. 298-302.

108. Furmaga, H., A. Shah, and A. Frazer, Serotonergic and noradrenergic pathways are required for the anxiolytic-like and antidepressant-like behavioral effects of repeated vagal nerve stimulation in rats. Biol Psychiatry, 2011. 70(10): p. 937-945.

109. Krahl, S.E., S.S. Senanayake, A.E. Pekary, and A. Sattin, Vagus nerve stimulation (VNS) is effective in a rat model of antidepressant action. J Psychiatr Res, 2004. 38(3): p. 237-240.

110. Grimonprez, A., R. Raedt, C. Baeken, P. Boon, and K. Vonck, The antidepressant mechanism of action of vagus nerve stimulation: Evidence from preclinical studies. Neurosci Biobehav Rev, 2015. 56: p. 26-34.

111. Duman, R.S., J. Malberg, and S. Nakagawa, Regulation of adult neurogenesis by psychotropic drugs and stress. J Pharmacol Exp Ther, 2001. 299(2): p. 401-407.

112. Duman, R.S., S. Nakagawa, and J. Malberg, Regulation of adult neurogenesis by antidepressant treatment. Neuropsychopharmacology, 2001. 25(6): p. 836-844.

113. Jacobs, B.L., H. van Praag, and F.H. Gage, Adult brain neurogenesis and psychiatry: a novel theory of depression. Mol Psychiatry, 2000. 5(3): p. 262-269.

114. Biggio, F., G. Gorini, C. Utzeri, P. Olla, F. Marrosu, et al., Chronic vagus nerve stimulation induces neuronal plasticity in the rat hippocampus. Int J Neuropsychopharmacol, 2009. 12(9): p. 12092021

115. Revesz, D., M. Tjernstrom, E. Ben-Menachem, and T. Thorlin, Effects of vagus nerve stimulation on rat hippocampal progenitor proliferation. Exp Neurol, 2008. 214(2): p. 259-265.

116. Gebhardt, N., K.J. Bar, M.K. Boettger, G. Grecksch, G. Keilhoff, et al., Vagus nerve stimulation ameliorated deficits in one-way active avoidance learning and stimulated hippocampal neurogenesis in bulbectomized rats. Brain Stimul, 2013. 6(1): p. 78-83.

117. Madsen, T.M., S.S. Newton, M.E. Eaton, D.S. Russell, and R.S. Duman, Chronic electroconvulsive seizure up-regulates beta-catenin expression in rat hippocampus: role in adult neurogenesis. Biol Psychiatry, 2003. 54(10): p. 1006-1014.

118. Hanson, N.D., C.B. Nemeroff, and M.J. Owens, Lithium, but not fluoxetine or the corticotropin-releasing factor receptor 1 receptor antagonist R121919, increases cell proliferation in the adult dentate gyrus. J Pharmacol Exp Ther, 2011. 337(1): p. 180-186.

119. Santarelli, L., M. Saxe, C. Gross, A. Surget, F. Battaglia, et al., Requirement of hippocampal neurogenesis for the behavioral effects of antidepressants. Science, 2003. 301(5634): p. 805-809.
120. Wainwright, S.R. and L.A. Galea, The neural plasticity theory of depression: assessing the roles of adult neurogenesis and PSANCAM within the hippocampus. Neural Plast, 2013. 2013: p. 805497.

121. Warner-Schmidt, J.L. and R.S. Duman, Hippocampal neurogenesis: opposing effects of stress and antidepressant treatment. Hippocampus, 2006. 16(3): p. 239-249.

122. Couillard-Despres, S., B. Iglseder, and L. Aigner, Neurogenesis, cellular plasticity and cognition: the impact of stem cells in the adult and aging brain-a mini-review. Gerontology, 2011. 57(6): p. 559-564.

123. Couillard-Despres, S., B. Winner, S. Schaubeck, R. Aigner, M. Vroemen, et al., Doublecortin expression levels in adult brain reflect neurogenesis. Eur J Neurosci, 2005. 21(1): p. 1-14.

124. Wang, J.W., D.J. David, J.E. Monckton, F. Battaglia, and R. Hen, Chronic fluoxetine stimulates maturation and synaptic plasticity of adult-born hippocampal granule cells. J Neurosci, 2008. 28(6): p. 1374-1384.

125. Yuan, T.F., A. Li, X. Sun, O. Arias-Carrion, and S. Machado, Vagus nerve stimulation in treating depression: A tale of two stories. Curr Mol Med, 2016. 16(1): p. 33-39.

126. Duman, R.S. and L.M. Monteggia, A neurotrophic model for stress-related mood disorders. Biol Psychiatry, 2006. 59(12): p. 1116-1127.

127. Nibuya, M., S. Morinobu, and R.S. Duman, Regulation of BDNF and trkB mRNA in rat brain by chronic electroconvulsive seizure and antidepressant drug treatments. J Neurosci, 1995. 15(11): p. 7539-7547.

128. Nibuya, M., E.J. Nestler, and R.S. Duman, Chronic antidepressant administration increases the expression of cAMP response element binding protein (CREB) in rat hippocampus. J Neurosci, 1996. 16(7): p. 2365-2372.

129. Furmaga, H., F.R. Carreno, and A. Frazer, Vagal nerve stimulation rapidly activates brain-derived neurotrophic factor receptor TrkB in rat brain. PLoS ONE, 2012. 7(5): p. e34844.

130. Shah, A.P., F.R. Carreno, H. Wu, Y.A. Chung, and A. Frazer, Role of TrkB in the anxiolytic-like and antidepressant-like effects of vagal nerve stimulation: Comparison with desipramine. Neuroscience, 2016. 322: p. 273-286.

131. Rantamaki, T., P. Hendolin, A. Kankaanpaa, J. Mijatovic, P. Piepponen, et al., Pharmacologically diverse antidepressants rapidly activate brain-derived neurotrophic factor receptor TrkB and induce phospholipase-Cgamma signaling pathways in mouse brain. Neuropsychopharmacology, 2007. 32(10): p. 2152-2162.

132. Saarelainen, T., P. Hendolin, G. Lucas, E. Koponen, M. Sairanen, et al., Activation of the TrkB neurotrophin receptor is induced by antidepressant drugs and is required for antidepressant-induced behavioral effects. J Neurosci, 2003. 23(1): p. 349-357.

133. Carreno, F.R. and A. Frazer, Activation of signaling pathways downstream of the brain-derived neurotrophic factor receptor, TrkB, in the rat brain by vagal nerve stimulation and antidepressant drugs. Int J Neuropsychopharmacol, 2014. 17(2): p. 247-258.

134. Li, S., X. Zhai, P. Rong, M.F. McCabe, J. Zhao, et al., Transcutaneous auricular vagus nerve stimulation triggers melatonin secretion and is antidepressive in Zucker diabetic fatty rats. PLoS One, 2014. 9(10): p. e111100.

135. Liu, R.P., J.L. Fang, P.J. Rong, Y. Zhao, H. Meng, et al., Effects of electroacupuncture at auricular concha region on the depressive status of unpredictable chronic mild stress rat models. Evid Based Complement Alternat Med, 2013. 2013: p. 789674.

136. Kitamura, Y., K. Akiyama, S. Hashimoto, K. Kitagawa, H. Kawasaki, et al., Effects of imipramine on extracellular serotonin and noradrenaline concentrations in ACTH-treated rats. Eur J Pharmacol, 2007. 566(1-3): p. 113-116.

137. Kitamura, Y., K. Akiyama, K. Kitagawa, K. Shibata, H. Kawasaki, et al., Chronic coadministration of carbamazepine together with 
imipramine produces antidepressant-like effects in an ACTH-induced animal model of treatment-resistant depression: involvement of 5-HT(2A) receptors? Pharmacol Biochem Behav, 2008. 89(3): p. 235-240.

138. Kitamura, Y., H. Araki, and Y. Gomita, Influence of ACTH on the effects of imipramine, desipramine and lithium on duration of immobility of rats in the forced swim test. Pharmacol Biochem Behav, 2002. 71(1-2): p. 63-69.
139. O'Leary, O.F., S. Zandy, T.G. Dinan, and J.F. Cryan, Lithium augmentation of the effects of desipramine in a mouse model of treatment-resistant depression: a role for hippocampal cell proliferation. Neuroscience, 2013. 228: p. 36-46.

140. Winter, C., B. Vollmayr, A. Djodari-Irani, J. Klein, and A. Sartorius, Pharmacological inhibition of the lateral habenula improves depressive-like behavior in an animal model of treatment resistant depression. Behav Brain Res, 2011. 216(1): p. 463-465. 